\title{
Doenças músculo-esqueléticas, trabalho e estilo de vida entre trabalhadores de uma instituição pública de saúde*
}

\author{
MUSKULOSKELETAL DISEASES, WORK AND LIFESTYLE AMONG PUBLIC WORKERS \\ ATAHEALTH INSTITUTION
}

\author{
ENFERMEDADES MÚSCULO-ESQUELÉTICAS, TRABAJO Y ESTILO DE VIDA \\ ENTRE TRABAJADORES DE UNA INSTITUCIÓN PÚBLICA DE SALUD
}

\section{Maria Silvia Monteiro', Neusa Maria Costa Alexandre², Cinthia Mendes Rodrigues ${ }^{3}$}

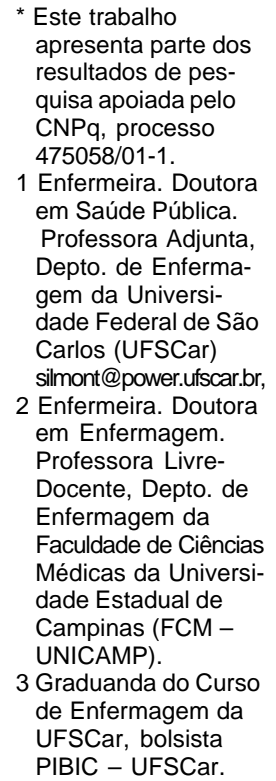

3 Graduanda do Curso de Enfermagem da UFSCar, bolsista PIBIC - UFSCar.

\section{RESUMO}

Foi desenvolvido um estudo transversal com 651 trabalhadores de diferentes ocupações. Os participantes responderam a um instrumento de coleta de dados gerais, ocupacionais e relativos ao estilo de vida, além do "Índice de Capacidade para o Trabalho", desenvolvido por pesquisadores finlandeses. Na análise dos fatores associados à ocorrência da doença, em estudos relativos às características sociodemográficas, foram identificados ser do gênero feminino, ter mais idade e ter baixa escolaridade. Em relação às características do trabalho, a associação à doença ocorreu naqueles com exigência predominantemente física, na maior duração semanal do segundo emprego, no maior tempo de trabalho na instituição. Quanto ao estilo de vida, estiveram associados à ocorrência de doença a obesidade, a longa duração das atividades domésticas e a não-realização de atividades de lazer.

\section{DESCRITORES}

Transtornos traumáticos cumulativos (epidemiologia). Estudos transversais. Trabalhadores.

\section{ABSTRACT}

This is a cross-sectional study developed with 651 workers of various occupations. They answered a questionnaire covering general, labor and lifestyle data, in addition to the Work Ability Index, which has been developed by researchers from Finland. In terms of sociodemographics, the analysis of the factors associated with the occurrence of musculoskeletal disorder revealed them to be female, older, and with low level of schooling. As for labor characteristics, the disease was associated with those individuals with physically demanding work, longer working hours on a second job and longer working hours at the institution itself. Regarding lifestyle, the characteristics associated with the occurrence of the disease were obesity, long housework hours and absence of leisure activities.

\section{KEY WORDS}

Cumulative trauma disorders (epidemiology). Cross-sectional study. Workers.

\section{RESUMEN}

Fue desarrollado un estudio transversal con 651 trabajadores de diferentes ocupaciones. Los participantes respondieron a un instrumento de recolección de datos generales, ocupacionales y relativos al estilo de vida, además del "Índice de Capacidad para el Trabajo", desarrollado por investigadores finlandeses. En el análisis de los factores asociados a la ocurrencia de la enfermedad, en estudios relativos a las características sociodemográficas, fueron identificados como del género femenino, tener más edad y baja escolaridad. En relación a las características del trabajo, la asociación a la enfermedad ocurrió en aquellos con exigencia predominantemente física, con mayor duración semanal en el segundo empleo, con el mayor tiempo de trabajo en la institución. En cuanto al estilo de vida, estuvieron asociados a la ocurrencia de enfermedad, la obesidad, la larga duración de las actividades domésticas y la no-realización de actividades de recreación.

\section{DESCRIPTORES}

Trastornos traumáticos acumulativos (epidemiología). Estudios transversales. Trabajadores. 


\section{INTRODUÇÃO}

As doenças músculo-esqueléticas são a maior causa de limitação funcional na população adulta em vários países ${ }^{(1-2)}$.

Na população trabalhadora brasileira os distúrbios osteomusculares relacionados ao trabalho figuram entre as doenças mais prevalentes, representando um relevante problema de saúde pública, que mereceu atenção do Ministério da Saúde por meio de duas publicações visando auxiliar os profissionais de saúde na prevenção, no diagnóstico, no tratamento e na reabilitação destas afecções ${ }^{(3-4)}$.

A literatura internacional tem destacado os trabalhadores da área de saúde como um grupo de risco para o desenvolvimento de distúrbios osteomusculares relacionados ao trabalho ${ }^{(5)}$.

Estudo desenvolvido com a população britânica para determinar a prevalência de dor na região cervical e sua relação com a ocupação e as atividades ocupacionais identificou a maior prevalência de sintomas entre trabalhadores da construção civil seguida pelas enfermeiras e pelos membros das forças armadas ${ }^{(6)}$.

No Brasil, estudos vêm sendo desenvolvidos enfocando distúrbios osteomusculares entre trabalhadores de enfermagem. Em estudos realizados em unidades de internação de um hospital que atende pacientes com alto grau de dependência, pesquisadores identificaram a prevalência de

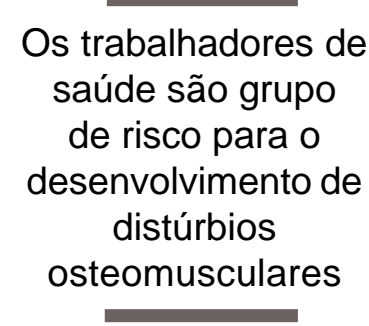

instituição de saúde localizada no interior do estado de São Paulo e que tem por objetivo a prestação de assistência à saúde na forma de moradia asilar.

Trata-se de um estudo epidemiológico transversal com 651 trabalhadores e que obteve uma taxa de resposta de $89.4 \%$. A população de estudo abrangeu todas as 29 ocupações existentes na instituição sendo que dez delas se referiam a trabalhadores de saúde.

Para a coleta de dados foram utilizados dois instrumentos: um questionário sobre dados gerais, ocupacionais e relativos ao estilo de vida e o "Índice de Capacidade para o Trabalho - ICT" ${ }^{{ }_{(9-10)}}$. O primeiro instrumento incluiu variáveis sócio-demográficas, variáveis relacionadas ao trabalho e ao estilo de vida. No segundo instrumento a fonte de dados das doenças músculo-esqueléticas foi o item do ICT, "Número Atual de Doenças Diagnosticadas por Médico", especificamente o item relativo às doenças em estudo. Este resgata a ocorrência destes eventos nos últimos doze meses, isto é, o número de vezes em que as doenças foram referidas. As doenças músculo-esqueléticas abrangem neste estudo: doenças da parte superior das costas ou região do pescoço, com dores freqüentes; doença da parte inferior das costas, com dores freqüentes; dor nas costas que se irradia para a perna (ciática); doença músculo-esquelética afetando os membros (braços e pernas), com dores frequientes; artrite reumatóide; e outra doença músculoesquelética, citando qual.

Os dados foram coletados no período de abril a junho de 2002. Após serem orientados, os indivíduos preencheram o instrumento de coleta de dados, que foi revisado por um dos autores. Aqueles que não sabiam ler, responderam ao questionário por meio de entrevista. Para inclusão na amostra, foram considerados todos os trabalhadores que compunham o quadro funcional da instituição no momento da coleta de dados. Foram excluídos os trabalhadores que estavam em afastamentos por licença no período de coleta de dados e os que se recusaram a participar. $\mathrm{O}$ projeto obteve a aprovação do Comitê de Ética da instituição e os participantes assinaram um termo de consentimento livre e esclarecido.

Os dados foram inseridos no Programa Epi-Info, versão 6.04d (Center for Diseases Control and Prevention, Atlanta, Georgia, USA).

Para este trabalho foi realizada uma análise descritiva dos dados dividido em dois grupos, os que referiram ou não a ocorrência de distúrbios músculo-esqueléticos com diagnóstico médico nos últimos doze meses, e a verificação de associação em relação às variáveis de estudo através do teste de associação pelo qui-quadrado, utilizando-se o referido programa.
O presente estudo apresenta parte dos resultados da pesquisa "Capacidade para o Trabalho e Envelhecimento entre Trabalhadores de uma Instituição Pública de Saúde", que recebeu apoio do CNPq. Este foi desenvolvido em uma 


\section{RESULTADOS E DISCUSSÃO}

A ocorrência de doença músculo-esquelética com diagnóstico médico foi referida 244 vezes.

Entre os indivíduos do gênero feminino a idade média foi de 43.1 anos e entre os do gênero masculino foi de 42.0 anos. Em relação às características sócio-demográficas (Tabela 1) a doença músculo-esquelética foi mais freqüente no gênero feminino $(\mathrm{p}=0.0266)$, apesar deste grupo ser mais jovem. A maior ocorrência em mulheres tem sido explicada pelas atividades do lar (dupla jornada); pelas ferramentas de trabalho inadequadas e pelas características funcionais desfavoráveis ${ }^{(11)}$.

A ocorrência de doença músculo-esquelética cresceu à medida que aumentou a idade $(\mathrm{p}=0.0000)$. Quanto à escolaridade, a doença em estudo foi mais freqüente no grupo de 5 a 8 anos de escolaridade (52.0\%), seguido pelos grupos com menor escolaridade $(\mathrm{p}=0.0017)$.

Tabela 1 - Doenças músculo-esqueléticas e as características sócio-demográficas - São Carlos, 2005

\begin{tabular}{|c|c|c|c|c|c|c|c|c|}
\hline \multirow[t]{3}{*}{ Variável } & \multirow[t]{3}{*}{ Categoria } & \multicolumn{6}{|c|}{ Doença M úsculo E squelética } & \multirow[b]{3}{*}{ Valor de $p$} \\
\hline & & \multicolumn{2}{|c|}{ Sim } & \multicolumn{2}{|c|}{ Não } & \multicolumn{2}{|c|}{ Total } & \\
\hline & & $\mathbf{N}$ & $\%$ & $\mathbf{N}$ & $\%$ & $\mathbf{N}$ & $\%$ & \\
\hline Gênero & $\begin{array}{l}\text { Feminino } \\
\text { Masculino }\end{array}$ & $\begin{array}{r}177 \\
67\end{array}$ & $\begin{array}{l}42,6 \\
28,4\end{array}$ & $\begin{array}{l}238 \\
169\end{array}$ & $\begin{array}{l}57,4 \\
71,6\end{array}$ & $\begin{array}{l}415 \\
236\end{array}$ & $\begin{array}{l}100,0 \\
100,0\end{array}$ & 0,0266 \\
\hline Grupo Etário & $\begin{array}{l}20-34 \\
35-44 \\
45-54 \\
55-69\end{array}$ & $\begin{array}{r}21 \\
49 \\
104 \\
70\end{array}$ & $\begin{array}{l}17,6 \\
28,5 \\
45,8 \\
52,6\end{array}$ & $\begin{array}{r}98 \\
123 \\
123 \\
63\end{array}$ & $\begin{array}{l}82,4 \\
71,5 \\
54,2 \\
47,4\end{array}$ & $\begin{array}{l}119 \\
172 \\
227 \\
133\end{array}$ & $\begin{array}{l}100,0 \\
100,0 \\
100,0 \\
100,0\end{array}$ & 0,0000 \\
\hline Escolaridade & $\begin{array}{l}\text { Nenhuma } \\
4 \text { anos } \\
8 \text { anos } \\
11 \text { anos } \\
\text { Curso universitário }\end{array}$ & $\begin{array}{l}10 \\
76 \\
51 \\
79 \\
28\end{array}$ & $\begin{array}{l}40,0 \\
46,6 \\
52,0 \\
29,6 \\
28,6\end{array}$ & $\begin{array}{r}15 \\
87 \\
47 \\
188 \\
70\end{array}$ & $\begin{array}{l}60,0 \\
53,4 \\
48,0 \\
70,4 \\
71,4\end{array}$ & $\begin{array}{r}25 \\
163 \\
98 \\
267 \\
98\end{array}$ & $\begin{array}{l}100,0 \\
100,0 \\
100,0 \\
100,0 \\
100,0\end{array}$ & 0,0017 \\
\hline
\end{tabular}

Quanto à relação entre a referência a doenças músculoesqueléticas e as características do trabalho (Tabela 2) observou-se que elas predominam entre os indivíduos que desenvolvem trabalhos com demandas predominantemente físicas $(52.7 \%$, valor de $\mathrm{p}=0.0000)$, que incluíam ocupações como auxiliares de serviço e oficiais de serviço de manutenção. A relação entre trabalho com exigências físicas e doença músculo-esquelética é um tema bastante estudado $^{(12)}$. Em trabalho desenvolvido na Escandinávia ${ }^{(13)}$ realizou-se o segmento de três projetos de intervenção em instituições de saúde visando verificar se a redução das horas diárias de trabalho levava à diminuição da ocorrência de distúrbios músculo-esqueléticos. A prevalência de dores no pescoço e nos ombros sofreu redução nos três estudos com a diminuição da duração diária do trabalho de $\geq 7$ horas para 6 horas, sendo que o mesmo não ocorreu com as dores nas costas.

No presente estudo os indivíduos de ocupações com exigência do trabalho física e mental (composta predominantemente por auxiliares de enfermagem) apresentaram $31.9 \%$ de ocorrência de distúrbios músculo-esqueléticos. Os fatores psicossociais e organizacionais têm cada vez mais assumido importante papel particularmente na evolução de queixas osteomusculares ${ }^{(11,14)}$.
Em estudo de intervenção realizado no Brasil ${ }^{(15)}$ foi desenvolvido um programa para redução de dores nas costas de auxiliares de enfermagem de um hospital universitário. Foram incluídos aqueles que apresentavam a queixa por no mínimo 6 meses e a intervenção foi conduzida durante 4 meses, duas vezes por semana em horário de trabalho e foi constituída de atividade educativa com abordagem ergonômica e a realização de exercícios, massagens e relaxamento. Os resultados apontaram para uma redução estatisticamente significante na freqüência de dor cervical nos últimos dois meses e na última semana no grupo de estudo.

A ocorrência das doenças em estudo cresceu com o aumento do tempo de trabalho na instituição, até a duração de 30 anos. A partir daí ela decresceu sugerindo que somente os mais saudáveis conseguem manter-se trabalhando (efeito do trabalhador sadio).

Não foram encontradas diferenças na frequiência de doenças músculo-esqueléticas entre os que possuem e os que não possuem um segundo emprego. Quando se analisa a duração semanal do segundo trabalho, observa-se maior ocorrência da doença em estudo entre os que trabalham de 31 a 40 horas $(\mathrm{p}=0.0014)$, e também que entre os que trabalham mais de 40 horas ocorre menor percentagem da doença, fato este sugestivo da ocorrência de efeito do trabalhador sadio. 
Entre as ocupações mais freqüentes na população de estudo ocorreram mais doenças músculo- esqueléticas entre os atendentes e os auxiliares de manutenção.

Tabela 2 - Doenças músculo-esqueléticas eas características do trabal ho - São Carlos, 2005

\begin{tabular}{|c|c|c|c|c|c|c|c|c|}
\hline \multirow[t]{3}{*}{ Variável } & \multirow[t]{3}{*}{ Categoria } & \multicolumn{6}{|c|}{ Doença Músculo Esquelética } & \multirow[b]{3}{*}{ Valor de $p$} \\
\hline & & \multicolumn{2}{|c|}{ Sim } & \multicolumn{2}{|c|}{ Não } & \multicolumn{2}{|c|}{ Total } & \\
\hline & & $\mathbf{N}$ & $\%$ & $\mathbf{N}$ & $\%$ & $\mathbf{N}$ & $\%$ & \\
\hline \multirow[t]{3}{*}{ Classe de demanda } & Exigência física & 116 & 52,7 & 104 & 47,3 & 220 & 100,0 & \multirow[t]{3}{*}{0,0000} \\
\hline & Exigência Mental & 30 & 24,2 & 94 & 75,8 & 124 & 100,0 & \\
\hline & Exigência mista & 98 & 31,9 & 209 & 68,1 & 307 & 100,0 & \\
\hline \multirow[t]{5}{*}{ Tempo de trabalho* } & Até 1 & 10 & 10,7 & 83 & 89,3 & 93 & 100,0 & \multirow[t]{5}{*}{0,0000} \\
\hline & 2 a 10 & 65 & 34,0 & 126 & 66,0 & 191 & 100,0 & \\
\hline & 11 a 20 & 61 & 37,9 & 100 & 62,1 & 161 & 100,0 & \\
\hline & 21 a 30 & 103 & 57,2 & 77 & 42,8 & 180 & 100,0 & \\
\hline & 31 a 50 & 5 & 19,2 & 21 & 80,8 & 26 & 100,0 & \\
\hline \multirow[t]{2}{*}{ Ter outro emprego } & Sim & 65 & 35,3 & 119 & 64,7 & 184 & 100,0 & \\
\hline & Não & 179 & 38,3 & 288 & 61,7 & 467 & 100,0 & \\
\hline \multirow{4}{*}{$\begin{array}{l}\text { Duração do outro } \\
\text { emprego }\end{array}$} & 1 a 20 & 27 & 37,5 & 45 & 62,5 & 72 & 100,0 & \multirow[t]{4}{*}{0,0014} \\
\hline & 21 a 30 & 11 & 32,3 & 23 & 67,7 & 34 & 100,0 & \\
\hline & 31 a 40 & 13 & 43,3 & 17 & 56,7 & 30 & 100,0 & \\
\hline & 41 a 50 & 5 & 17,9 & 23 & 82,1 & 28 & 100,0 & \\
\hline \multirow{4}{*}{$\begin{array}{l}\text { Ocupações mais } \\
\text { frequentes }\end{array}$} & Aux. de enfermagem & 73 & 29,2 & 177 & 70,8 & 250 & 100,0 & \multirow[t]{4}{*}{0,0001} \\
\hline & Aux. de serviços gerais & 88 & 52,1 & 81 & 47,9 & 169 & 100,0 & \\
\hline & Aux. de manutenção & 24 & 53,3 & 21 & 46,7 & 45 & 100,0 & \\
\hline & Atendente & 18 & 58,1 & 13 & 41,9 & 31 & 100,0 & \\
\hline
\end{tabular}

* em anos.

A análise da ocorrência de doenças músculoesqueléticas em relação às características do estilo de vida (Tabela 3) não mostrou diferença entre os fumantes e nãofumantes. Entre os fumantes que consomem de 10 a 20 cigarros por dia $46.5 \%$ referiram a doença em estudo $(\mathrm{p}=0.0438)$.

Em relação ao índice de massa corpórea, os obesos apresentam alta porcentagem de indivíduos com a doença, $68.0 \%$ $(\mathrm{p}=0.0000)$. Apesar da literatura apresentar dados controversos sobre o relacionamento de características pessoais e sintomas osteomusculares, a obesidade parece ser um risco particularmente para a coluna lombar ${ }^{(16)}$.

A doença foi mais frequente entre os que realizam atividade doméstica $(\mathrm{p}=0.0235)$ e entre os que o fazem por mais tempo $(\mathrm{p}=0.0106)$.
A prática de atividade física foi referida similarmente pelos doentes e não-doentes, assim como a duração semanal da mesma.

Quando se considera os tipos de atividades física mais frequentes, a maior porcentagem de referências à doença ocorreu entre os que realizam caminhada ( $\mathrm{p}=0.0496)$, possivelmente porque as outras atividades físicas mais referidas, a prática de ginástica, futebol ou vôlei, tem maior grau de exigência física e só podem ser praticadas por indivíduos cuja doença não cause impedimento significativo.

A realização de atividade de lazer foi referida por aproximadamente um terço dos que relataram a ocorrência de doença músculo-esquelética $(\mathrm{p}=0.0005)$, sugerindo repercussões sobre a qualidade de vida dos trabalhadores afetados. 
Tabela 3- Doenças músculo-esqueléticas eas características deestilo de vida - São Carlos, 2005

\begin{tabular}{|c|c|c|c|c|c|c|c|c|}
\hline \multirow[t]{3}{*}{ Variável } & \multirow[t]{3}{*}{ Categoria } & \multicolumn{6}{|c|}{ Doença Músculo Esquelética } & \multirow[b]{3}{*}{ Valor de $p$} \\
\hline & & \multicolumn{2}{|c|}{ Sim } & \multicolumn{2}{|c|}{ Não } & \multicolumn{2}{|c|}{ Total } & \\
\hline & & $\mathbf{N}$ & $\%$ & $\mathbf{N}$ & $\%$ & $\mathbf{N}$ & $\%$ & \\
\hline \multirow[t]{2}{*}{ Hábito de fumar } & Sim & 116 & 38,9 & 182 & 61,1 & 298 & 100,0 & \\
\hline & Não & 128 & 36,3 & 225 & 63,7 & 353 & 100,0 & \\
\hline \multirow{3}{*}{$\begin{array}{l}\text { Número de cigarros por } \\
\text { dia }\end{array}$} & 1 a 10 & 25 & 29,8 & 59 & 70,2 & 84 & 100,0 & 0,0438 \\
\hline & 11 a 20 & 33 & 46,5 & 38 & 53,5 & 71 & 100,0 & \\
\hline & 21 a 50 & 3 & 33,3 & 6 & 66,7 & 9 & 100,0 & \\
\hline \multirow{3}{*}{$\begin{array}{l}\text { Í ndice de massa } \\
\text { corpórea* }\end{array}$} & Normal & 91 & 27,8 & 236 & 72,2 & 327 & 100,0 & 0,0000 \\
\hline & Sobrepeso & 91 & 40,8 & 132 & 59,2 & 223 & 100,0 & \\
\hline & Obesidade & 53 & 68,0 & 25 & 32,0 & 78 & 100,0 & \\
\hline \multirow{2}{*}{$\begin{array}{l}\text { Realização de atividade } \\
\text { doméstica }\end{array}$} & Sim & 216 & 40,1 & 322 & 59,9 & 538 & 100,0 & 0,0235 \\
\hline & Não & 28 & 24,8 & 85 & 75,2 & 113 & 100,0 & \\
\hline \multirow{4}{*}{$\begin{array}{l}\text { Duração da atividade } \\
\text { doméstica** }\end{array}$} & 1 a 20 & 71 & 24,1 & 224 & 75,9 & 295 & 100,0 & 0,0106 \\
\hline & 21 a 40 & 49 & 26,8 & 134 & 73,2 & 183 & 100,0 & \\
\hline & 41 a 60 & 44 & 35,8 & 79 & 64,2 & 123 & 100,0 & \\
\hline & $>60$ & 12 & 44,4 & 15 & 55,6 & 27 & 100,0 & \\
\hline \multirow{2}{*}{$\begin{array}{l}\text { Prática de atividade } \\
\text { física }\end{array}$} & Sim & 101 & 34,8 & 189 & 65,2 & 290 & 100,0 & \\
\hline & Não & 143 & 39,6 & 218 & 60,4 & 361 & 100,0 & \\
\hline \multirow{3}{*}{$\begin{array}{l}\text { Duração da atividade } \\
\text { física*** }\end{array}$} & Até 180 & 43 & 32,8 & 88 & 67,2 & 131 & 100,0 & \\
\hline & 181 a 360 & 30 & 33,3 & 60 & 66,7 & 90 & 100,0 & \\
\hline & $>360$ & 14 & 37,8 & 23 & 62,2 & 37 & 100,0 & \\
\hline \multirow[t]{3}{*}{ Tipo de atividade física } & Caminhada & 53 & 37,9 & 87 & 62,1 & 140 & 100,0 & 0,0496 \\
\hline & Ginástica & 12 & 23,5 & 39 & 76,5 & 51 & 100,0 & \\
\hline & Futebol-vôlei & 9 & 26,5 & 25 & 73,5 & 34 & 100,0 & \\
\hline \multirow{2}{*}{$\begin{array}{l}\text { Realização de atividade } \\
\text { de lazer }\end{array}$} & Sim & 227 & 36,4 & 396 & 63,6 & 623 & 100,0 & 0,0005 \\
\hline & Não & 17 & 60,7 & 11 & 39,3 & 28 & 100,0 & \\
\hline
\end{tabular}

* sendo normal $\leq 24.8$, sobrepeso $>24.8$ e $\leq 29.3$ e obesidade $>29.3$

** em horas por semana

*** em minutos por semana

\section{CONCLUSÃO}

A análise dos fatores associados à ocorrência de doença músculo-esquelética permitiu identificar fatores relativos ao trabalho que podem subsidiar o desenvolvimento de ações preventivas, curativas e de reabilitação em relação aos grupos mais vulneráveis: aqueles que desenvolvem ocupações com trabalhos de exigência predominantemente física e aqueles com menor grau de escolaridade. Outros aspectos, como ter um segundo emprego e com longa duração semanal da carga horária são mais complexos, pois decorrem das condições de vida.

\section{REFERÊNCIAS}

(1) Woolf AD, Pfleger B. Burden of major muskuloskeletal conditions. Bull World Health Organ. 2003;81(9):646-56.
O mesmo acontece em relação às características do estilo de vida que se mostraram associadas à ocorrência de doença músculo-esquelética: algumas, como a obesidade, podem desencadear ações visando o alcance de peso normal por meio de ações educativas, enquanto outras, como a longa duração semanal das atividades domésticas, dependem das condições de vida.

Mas certamente a intervenção no ambiente de trabalho visando a redução da carga de trabalho físico pode ter efeitos positivos tanto sobre a ocorrência de doenças músculoesqueléticas, como sobre a qualidade de vida dos indivíduos por ela afetados.
(2) Yelin E. Cost of musculoskeletal diseases: impact of work disability and functional decline. J Rheumatol. 2003;30(Sup 68):8-11.

$\begin{aligned} \text { Rev Esc Enferm USP } & \text { Doenças músculo-esqueléticas, trabalho e estilo de vida entre } \\ 2006 ; 40(1): 20-5 . & \text { trabalhadores de uma instituição pública de saúde } \\ \text { Mww.ee.usp.br/reeusp/ } & \text { Monteiro MS, Alexandre NMC, Rodrigues CM. }\end{aligned}$


(3) Brasil. Ministério da Saúde. Diagnóstico, tratamento, reabilitação, prevenção e fisiopatologia das LER/DORT. Brasília; 2001. (Série A. Normas e Manuais Técnicos, 105).

(4) Brasil. Ministério da Saúde. LER/DORT: dilemas, polêmicas e dúvidas. Brasília; 2001. (Série A. Normas e Manuais Técnicos, 104).

(5) Fragala G, Bailey LP. Adressing occupational strains and sprains. Musculoskeletal injuries in hospitals. AAOHN J. 2003;51 (6):253-9.

(6) Palmer KT, Walker-Bone K, Griffin MJ, Syddall H, Pannett B, Coggon D, et al. Prevalence and occupational associations of neck pain in the British population. Scand J Work Environ Health. 2001;27(1):49-56.

(7) Gurgueira GP, Alexandre NMC, Corrêa Filho HR. Prevalência de sintomas músculo-esqueléticos em trabalhadoras de enfermagem. Rev Lat Am Enferm. 2003;11(5):608-13.

(8) Alexandre NMC, Angerami ELS, Moreira Filho DC. Dores nas costas e enfermagem. Rev Esc Enferm USP. 1996;30(2): 267-85.

(9) Tuomi K, Ilmarinen J, Jahkola A, Katajarinne L. Índice de capacidade para o trabalho. Helsinki: Finnish Institute of Occupational Health; 1997.
(10) Tuomi K, Ilmarinen J, Jahkola A, Katajarinne L. Work ability index. $2^{\text {nd }}$ ed. Helsinki: Finish Institute of Occupational Health; 1998.

(11) Malchaire J, Cock N, Vergracht S. Review of the factors associated with musculoskeletal problems in epidemiological studies. Int Arch Occup Environ Health. 2001;74(2):79-90.

(12) National Institute for Occupational Safety and Health (NIOSH). Musculoskeletal disorders and workplace factors. Cincinnati: NIOSH; 1997.

(13) Wergeland ED, Veiersted B, Ingre M, Olsson B, Åkerstedt I, Bjørnskau T, et al.. A shorter workday as a means of reducing the occurrence of musculoskeletal disorders. Scand J Work Environ Health. 2003;29(1):27-34.

(14) Nahit ES, Hunt IM, Lunt M, Dunn G, Silman AJ, Macfarlane GJ. Effects of psychosocial and individual psychological factors on the onset of musculoskeletal pain: common and site-specific effects. Ann Rheum Dis. 2003;62(8):755-60.

(15) Alexandre NMC, Moraes MAA, Corrêa Filho HR. Evaluation of a program to reduce back pain in nursing personnel. Rev Saúde Pública. 2001;35(4):356-61.

(16) Hales TR, Bernard BP. Epidemiology of work-related musculoskeletal disorders. Orthop Clin North Am. 1996;27(4):679-709.

\section{AGRADECIMENTO}

Agradecemos à FAPESP pelo apoio ao desenvolvimento deste trabalho e ao CNPq pelo apoio à pesquisa original da qual este estudo derivou 